\title{
Caracterização física de frutos e sementes de Lafoensia pacari, Alibertia edulis e Genipa americana
}

\section{Physical characterization of fruits and seeds of Lafoensia pacari, Alibertia edulis and Genipa americana}

\author{
Severino de Paiva Sobrinho1, ${ }^{1,}$, Maria Cristina de Figueiredo e Albuquerque ${ }^{2}$, Petterson \\ Baptista da Luz $^{1}$ e Elisangela Clarete Camili²
}

\author{
${ }^{1}$ Curso de Agronomia, Faculdade de Ciências Agrárias e Biológicas, Universidade do Estado de Mato Grosso, Cidade Universitária, S/N, Caixa Postal, 118: 78.200-970. Cáceres-MT, Brasil \\ 2 Departamento de Fitotecnia, Faculdade de Agronomia, Medicina Veterinária e Zootecnia, Universidade Federal de Mato Grosso, Av. Fernando Corrêa da Costa No 2367, Boa esperança, 78060- \\ -900. Cuiabá - MT, Brasil \\ (*E-mail paivasevero@unemat.br) \\ http://dx.doi.org/10.19084/RCA16034
}

Recebido/received: 2016.03.14

Recebido em versão revista/received in revised form: 2016.11.10

Aceite/accepted: 2016.11.17

\section{R E S U M O}

Como a biometria dos frutos e sementes fornece informações para a conservação e exploração da espécie, de variabilidade entre indivíduos numa determinada área e permite comparações de uma mesma espécie que ocorre em localidades geográficas diferentes, objetivou-se, neste trabalho, caracterizar biometricamente frutos e sementes de Lafoensia pacari, Alibertia edulis e Genipa americana. Para isso foram selecionados aleatoriamente 60 frutos e número igual de sementes, os quais foram medidos com o auxílio de craveira digital. Nos frutos de L. pacari foram medidos comprimento, largura e espessura e nos de A. edulis e G. americana diâmetro transversal e longitudinal; nas sementes determinou-se comprimento, largura e espessura. Também foram determinados o número de sementes por fruto e a massa da matéria fresca dos frutos e sementes. O número de sementes por fruto e a massa da matéria fresca das sementes são as características biométricas com maior variação. O coeficiente de assimetria é negativo para a maioria das características biométricas do fruto e positivo para a maioria das características das sementes. O coeficiente de curtose, para todas as características biométricas dos frutos e sementes apresenta valor menor que 3, exceto para comprimento do fruto de L. pacari.

Palavras-chave: cerrado, biometria, espécies florestais.

\section{A B S T R A C T}

Biometry of fruits and seeds provides information for conservation and species exploitation, the variability among individuals in a determined area and allows comparisons on the same species occurring in different geographic locations. Thus, the aim of this study was to characterize biometrically fruits and seeds from Lafoensia pacari, Alibertia edulis and Genipa americana. Sixty fruits and an equal number of seeds were randomly selected and measured with the aid of digital calipers. The length, width and thickness of $L$. pacari fruits was measured, while the transverse and longitudinal diameter was measured in A. edulis and G. americana; in the seeds, the length, width and thickness were measured. It was also determined the number of seeds per fruit and fresh matter mass of fruits and seeds. The number of seeds per fruit and fresh matter mass of seeds are the biometric characteristics with greater variation. The asymmetry coefficient is negative for most fruit biometric characteristics and positive for most seeds characteristics. The kurtosis coefficient, for all biometric characteristics of fruits and seeds, shows value less than three, except for fruit length of L. pacari.

Keywords: cerrado, biometrics, forest species. 


\section{INTRODUÇÃO}

O cerrado é muito promissor em termos de biodiversidade de árvores frutíferas (Almeida et al., 1987), sendo considerado uma das mais ricas savanas do mundo, apresentando uma flora com mais de 11627 espécies vasculares (Mendonça et al., 2008), o que soma aproximadamente $30 \%$ da biodiversidade brasileira e $5 \%$ da mundial. A redução das áreas de matas nativas no cerrado vem pondo em risco esta diversidade, entre elas diversas espécies vegetais, que apresentam potencial fármaco, madeireiro e alimentício, como mangava brava (Lafoensia pacari St. Hil.), marmelada bola (Alibertia edulis (L.C.Rich.) A.Rich. ex DC.) e jenipapo (Genipa americana L.).

Muitas espécies do cerrado não foram ainda estudadas, e dessa forma pouco se conhece sobre o potencial de exploração das mesmas. Na busca de conhecimentos que possam fornecer informações sobre essas espécies vegetais e auxiliar na sua identificação e classificação, na produção de sementes, nos estudos de sucessão ecológica e na regeneração dos ecossistemas florestais, destaca-se a caracterização biométrica de frutos e sementes.

Pesquisas referentes a caracteres morfológicos de frutos, sementes e desenvolvimento de plântulas e plantas jovens são frequentes para diversas espécies (Moura et al., 2013). Para as espécies não domesticadas, esse tipo de estudo tem sido importante para identificar o potencial de uso das mesmas no melhoramento genético. A biometria dos frutos fornece informações para a conservação e exploração dos recursos de valor econômico, permitindo um incremento contínuo da busca racional e uso eficaz dos frutos (Gusmão et al., 2006). Também a biometria é um importante instrumento para detectar a variabilidade genética dentro de populações de uma mesma espécie, e as relações entre esta variabilidade e os fatores ambientais, em programas de melhoramento genético (Vieira e Gusmão, 2008).

Em sementes, a importância da biometria ocorre pelo fato da mesma se relacionar com as características de dispersão e pode ser utilizada para distinguir espécies pioneiras das não pioneiras (Baskin e Baskin, 1998). Outra relevância da biometria de frutos e sementes, bem como o conhecimento da morfologia e desenvolvimento das plântulas, é o contributo para estudos de germinação e produção de mudas auxiliando técnicas de cultivo para as espécies (Battilani et al., 2011).

Diante do exposto, no presente trabalho o objetivo foi caracterizar fisicamente frutos e sementes de três espécies provenientes do cerrado, L. pacari, A. edulis e G. americana, para estabelecer estratégias de identificação, preservação e utilização da variabilidade genética das espécies.

\section{MATERIAL E MÉTODOS}

Os frutos de L. pacari, A. edulis e G. americana foram colhidos em três locais (Peraputanga: $16^{\circ} 03^{\prime} 35^{\prime \prime} \mathrm{S}$ e $57^{\circ} 33^{\prime} 60^{\prime \prime} \mathrm{W}$, Vila Aparecida: $15^{\circ} 48^{\prime} 25^{\prime \prime} \mathrm{S}$ e $57^{\circ} 27^{\prime} 27^{\prime} \mathrm{W}$ e perímetro urbano: $16^{\circ} 03^{\prime} 15^{\prime \prime}$ S e $57^{\circ} 40^{\prime} 04^{\prime \prime} \mathrm{W}$ ) do município de Cáceres-MT, nos meses de agosto, setembro e novembro de 2012, respectivamente. Para cada espécie, os frutos foram colhidos em 10 matrizes, a uma distância de pelo menos 50 metros entre as mesmas, e em cada uma delas foram colhidos 15 frutos, totalizando 150 frutos por espécie.

A colheita dos frutos de L. pacari e A. edulis foi realizada diretamente na planta, entretanto os frutos de A. edulis foram colhidos sem a completa maturação, sendo a mesma finalizada no laboratório, enquanto os frutos de G. americana foram colhidos completamente maduros no solo sob a copa das árvores.

Para avaliar as caraterísticas biométricas dos frutos foi retirada uma amostra aleatória de 60 frutos do total de cada espécie. As características avaliadas em L. pacari foram: massa da matéria fresca (MFF), comprimento $(\mathrm{CF})$, largura (LF), espessura (EF) e número de sementes (NSF); para $A$. edulis e G. americana foram: massa da matéria fresca, diâmetro longitudinal (DLF) e transversal (DTF) e número de sementes.

Após a avaliação dos frutos de A. edulis e G. americana, os mesmos foram abertos manualmente e as sementes com a polpa foram colocadas sobre uma peneira e lavadas em água corrente para remover toda a polpa; as sementes de L. pacari foram removidas manualmente. Depois da remoção, as sementes foram deixadas sobre papel absorvente 
na bancada do laboratório durante 48 horas. Após esse período, foi retirada uma amostra aleatória de 60 sementes de cada espécie para determinar: a massa (MFS), o comprimento (CS), a largura (LS) e a espessura (ES). As determinações das dimensões de frutos e sementes foram feitas com auxílio de uma craveira digital e para matéria fresca foi utilizada balança analítica com precisão de 0,0001 g. Para determinação do teor de água, em cada espécie foram retiradas duas subamostras de 25 sementes, pesadas e levadas para estufa a $105 \pm 3^{\circ} \mathrm{C}$ e mantidas por 24 horas (Brasil, 2009). Para estimar a massa de 1000 sementes foram utilizadas oito amostras de 100 sementes conforme recomendação de Brasil (2009).

Em seguida os dados foram submetidos à análise estatística para determinação da média, desvio padrão, coeficiente de variação, assimetria e curtose, valor máximo e mínimo e correlação de Pearson. Os valores de referência adotados para o coeficiente de assimetria foram: $S<0$, distribuição assimétrica a esquerda e $S>0$, distribuição assimétrica a direita e para o coeficiente de curtose foram: $K>3$, distribuição mais "afilada" que a normal (leptocúrtica) e $K<3$, distribuição mais achatada que a normal (platicúrtica). Na avaliação de correlações entre variáveis, consideraram-se as seguintes classes de correlação: forte $(0,8 \leq \mathrm{P}<1)$, moderada $(0,5 \leq \mathrm{P}<0,8)$, fraca $(0,1 \leq \mathrm{P}<0,5)$ e ínfima $(0<P<0,1)$ conforme Santos (2010). Para as análises foi utilizado o programa estatístico $R$ versão 2.15 .2 (R Core Team, 2012).

\section{RESULTADOS E DISCUSSÃO}

O fruto de L. pacari é seco, deiscente, semigloboso e possui ápice arredondado, terminando em cone. As sementes são de coloração pardo-amarelada, planas, com presença de alas bilaterais que servem para dispersão anemocórica. Em A. edulis os frutos são do tipo bacóide, formato arredondado, casca lisa de coloração verde, tornando-se pardo-clara quando maduros; as sementes são pequenas com pouca espessura e formato irregular, com coloração também pardo-clara. Para a espécie G. americana, os frutos são do tipo baga com formato subgloboso, de cor parda quando maduros e casca mole, as sementes são de cor parda e formato achatado. As sementes analisadas tinham teor de água de
$10,2 \%$ (L. pacari), $12,3 \%$ (A. edulis) e $14,4 \%$ (G. americana). A massa de 1000 sementes foi igual a 30,13, 14,60 e 40,37 g, para L. pacari, A. edulis e G. americana, respectivamente.

Para os frutos das três espécies em estudo, o maior desvio padrão foi verificado para a característica número de sementes por fruto; quanto ao coeficiente de variação, também se observou o mesmo resultado, exceto para L. pacari, em que a característica biométrica com maior coeficiente de variação foi a massa da matéria fresca do fruto (Quadro 1). Para as sementes, a massa da matéria fresca foi a característica que apresentou maior desvio padrão e coeficiente de variação, isso para as três espécies em estudo.

$\mathrm{Na}$ avaliação da variabilidade de características biométricas, segundo Moura et al. (2010), quanto maior for o coeficiente de variação de uma determinada característica, maior será a possibilidade de seleção. Essas variações nas características biométricas podem ser promovidas tanto por fatores ambientais durante a floração e o desenvolvimento, como também pode representar um indício de alta variabilidade genética da população (Sangalli et al., 2012).

Os frutos de $L$. pacari apresentaram, em média, 27,51, 31,09 e 29,33 mm de comprimento, espessura e largura, respectivamente, enquanto a massa da matéria fresca foi de $8,84 \mathrm{~g}$ e o número de sementes por fruto foi de 125,83 (Quadro 1). Entre as características biométricas avaliadas na $L$. pacari, observou-se que a maior amplitude ocorreu para massa da matéria fresca, cujo valor máximo é cerca de cinco vezes superior ao valor mínimo.

Quanto ao comprimento dos frutos de L. pacari, o valor observado foi menor em relação aos avaliados por Silva Junior (2005), que verificou valores entre 40 e $80 \mathrm{~mm}$ e o número de sementes por fruto apresentou variação diferente da observada por esse autor, que variou de 15 a 190 sementes por fruto. A massa da matéria fresca dos frutos de $L$. pacari teve variação entre 3,57 e $18,36 \mathrm{~g}$, sendo esses limites diferentes da faixa $(6,1$ a $40,6 \mathrm{~g})$ descrita por Carvalho (2003a).

Em frutos de $A$. edulis, a massa da matéria fresca dos frutos apresentou valor médio de $46,77 \mathrm{~g}$ e a 
Quadro 1 - Valores mínimo (Min), máximo (Max), média, desvio padrão (DP), coeficiente de variação (CV), assimetria (S) e curtose (K) referentes a caracterização biométrica de frutos das três espécies colhidas no município de Cáceres/ MT. 2013

\begin{tabular}{|c|c|c|c|c|c|c|c|c|}
\hline & Características & Mín & Máx & Média & DP & CV (\%) & $S$ & K \\
\hline \multirow{5}{*}{ 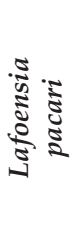 } & $\mathrm{CF}(\mathrm{mm})$ & 20,29 & 41,56 & 27,51 & 3,43 & 12,46 & 1,17 & 4,31 \\
\hline & $\mathrm{EF}(\mathrm{mm})$ & 23,54 & 38,80 & 31,09 & 3,76 & 12,01 & $-0,03$ & $-0,76$ \\
\hline & $\mathrm{LF}(\mathrm{mm})$ & 21,97 & 37,26 & 29,33 & 3,55 & 12,10 & $-0,05$ & $-0,32$ \\
\hline & MFF (g) & 3,57 & 18,36 & 8,84 & 3,56 & 40,34 & 0,94 & 0,64 \\
\hline & NSF & 100 & 187 & 125,83 & 20,61 & 16,38 & 0,98 & 0,52 \\
\hline \multirow{4}{*}{ 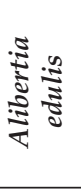 } & DLF (mm) & 32,55 & 47,46 & 39,36 & 3,09 & 7,85 & 0,09 & 0,05 \\
\hline & DTF (mm) & 34,60 & 52,45 & 44,61 & 4,23 & 9,48 & $-0,11$ & $-0,44$ \\
\hline & MFF (g) & 23,83 & 70,20 & 46,77 & 11,01 & 23,54 & 0,29 & $-0,34$ \\
\hline & NSF & 20 & 338 & 169,88 & 75,45 & 44,41 & 0,14 & $-0,79$ \\
\hline \multirow{4}{*}{ 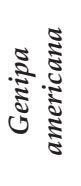 } & DLF (mm) & 52,79 & 88,23 & 74,40 & 8,97 & 12,05 & $-0,45$ & $-0,75$ \\
\hline & DTF (mm) & 52,13 & 75,78 & 64,47 & 5,62 & 8,70 & $-0,31$ & $-0,47$ \\
\hline & MFF (g) & 60,16 & 221,95 & 150,13 & 37,44 & 24,94 & $-0,51$ & $-0,25$ \\
\hline & NSF & 39,00 & 341,00 & 223,15 & 62,85 & 28,16 & $-0,49$ & 0,55 \\
\hline
\end{tabular}

$\mathrm{CF}=$ comprimento do fruto, $\mathrm{EF}=$ espessura do fruto, $\mathrm{LF}=$ largura do fruto, $\mathrm{MFF}=$ matéria fresca do fruto, $\mathrm{DLF}=$ diâmetro longitudinal do fruto, $\mathrm{DTF}=$ diâmetro transversal do fruto e NSF = número de sementes por fruto.

maior amplitude foi observada na característica número de sementes por fruto, onde o menor número foi 20 e o maior 338 (Quadro 1), o que equivale aproximadamente a 16 vezes, essa diferença. Observou-se que os frutos de $A$. edulis possuem diâmetro transversal maior do que o longitudinal, indicando frutos levemente achatados. A faixa correspondente a estes diâmetros é diferente da descrita por Martin et al. (1987), os quais descrevem uma faixa de 15 a $30 \mathrm{~mm}$ de diâmetro do fruto.

A maior variação entre o valor máximo e mínimo em G. americana ocorreu para a característica número de sementes por fruto, o qual ficou entre 39 e 341. Os diâmetros longitudinal e transversal apresentaram distribuição entre 52,79 e 88,23 e 52,13 e $75,78 \mathrm{~mm}$, respectivamente, quanto a massa da matéria fresca os menores frutos apresentaram 60,16 e os maiores 221,95 g (Quadro 1).

Os valores do diâmetro longitudinal apresentaram uma amplitude superior aos obtidos por Donadio etal. (1998), os quais obtiveram a faixa de 60 a $70 \mathrm{~mm}$, no entanto, verificou-se o oposto para o diâmetro transversal, onde os autores obtiveram os valores entre 80 a $100 \mathrm{~mm}$. Os frutos maiores apresentaram uma massa fresca cerca de quatro vezes superior aos menores; esta faixa foi menor do que a encontrada por Naves et al. (1995), que foi de 44 a $440 \mathrm{~g}$. A variação de valores foi grande para o número de sementes por fruto e maior do que a encontrada por Naves et al. (1995), que foi de 87 a 290 sementes por fruto. O número de sementes por fruto pode ser influenciado diretamente pelas condições ambientais e dentre estas condições a disponibilidade hídrica durante a floração (Marcos Filho, 2015).

As variações no tamanho dos frutos podem estar relacionadas com a diversidade genética da espécie na população, porém é possível que a modificação no tamanho do fruto não esteja apenas relacionada com o património genético, mas também com condições determinadas pelo ambiente (Nogueira et al., 2010) e de acordo com Silva Junior et al. (2012), a massa do fruto e o número de sementes por fruto são influenciados pela precipitação e fertilidade do solo, logo, estes valores podem variar de uma região para outra, bem como de um ano para outro. Segundo Araújo et al. (2004), informações sobre o tamanho do fruto poderão contribuir para o reconhecimento da espécie nos trabalhos de levantamento florístico.

O coeficiente de assimetria foi negativo para espessura e largura do fruto em L. pacari, como também para diâmetro transversal do fruto de $A$. edulis e para todas as características biométricas do fruto 
de G. americana, enquanto o coeficiente de curtose foi maior que três $(K>3)$ apenas para o comprimento dos frutos de L. pacari (Quadro 1).

Todas as variáveis biométricas dos frutos apresentaram distribuição platicúrtica, indicando que a distribuição de frequência das características analisadas é mais achatada do que a curva normal, exceto o comprimento de fruto de L. pacari que apresentou distribuição leptocúrtica, onde a distribuição de frequência é mais "afilada" do que a curva normal. Quando a distribuição é do tipo platicúrtica indica maior amplitude de distribuição e para o tipo leptocúrtica o oposto.

Em média as sementes de L. pacari apresentaram comprimento, largura e espessura igual a 18,70, 9,55 e 0,86 mm, enquanto a massa da matéria fresca teve média de 32,05 mg (Quadro 2), sendo este valor superior ao encontrado por Brüning et al. (2011), que foi de 18,05 mg.

As sementes de $A$. edulis apresentaram comprimento variando entre 3,67 e 6,29 $\mathrm{mm}$, largura de 2,19 a $4,15 \mathrm{~mm}$ e espessura de 1,06 a $2,39 \mathrm{~mm}$; em média, essas dimensões foram 5,01, 3,24 e 1,58 mm, respectivamente, e a massa da matéria fresca variou de 6,40 a 24,10 mg e média de 13,84 mg.

A análise biométrica das sementes de G. americana apresentou as seguintes variações: 5,53 a 9,53, 4,17 a 7,86 e 1,13 a 2,46 $\mathrm{mm}$ para comprimento, largura e espessura e 23,30 a $64,50 \mathrm{mg}$ para massa da matéria fresca das sementes, com médias de 7,11, 5,82 e 1,67 mm para comprimento, largura e espessura, e 37,57 mg para a massa da matéria fresca das sementes, valor este inferior ao obtido por Silva et al. (2001). O intervalo de variação do comprimento das sementes de G. americana foi inferior ao obtido por Carvalho (2003b), os quais variaram de 10 a $12 \mathrm{~mm}$, e o menor comprimento de semente no presente estudo foi $0,47 \mathrm{~mm}$, inferior ao obtido por Figueiredo et al. (1991).

Segundo Santos et al. (2009a), quando as matrizes se encontram em área com pouca variação macroambiental (como por exemplo temperatura e precipitação), as variações nas características advêm principalmente de efeitos genéticos e/ou microambientais. O conhecimento da variação biométrica de caracteres de sementes é importante para o melhoramento dessas características, seja no sentido de aumento ou uniformidade (Santos et al., $2009 b)$, pois sementes maiores tendem a apresentar maior conteúdo de reserva, o que lhes confere maior vigor e resistência no armazenamento, como também a uniformidade das sementes que facilita a seleção e a sementeira das mesmas.

O coeficiente de assimetria das características biométricas das sementes de L. pacari e G. americana, bem como espessura e massa da matéria fresca

Quadro 2 - Valores mínimo (Min), máximo (Max), média, desvio padrão (DP), coeficiente de variação (CV), assimetria (S) e curtose (K) referentes a caracterização biométrica de sementes de três espécies colhidas no município de Cáceres/ MT. 2013

\begin{tabular}{|c|c|c|c|c|c|c|c|c|}
\hline & Características & Mín & Máx & Média & DP & CV (\%) & $S$ & $\mathbf{K}$ \\
\hline \multirow{4}{*}{ 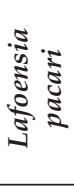 } & $\mathrm{CS}(\mathrm{mm})$ & 13,80 & 23,09 & 18,70 & 2,14 & 11,44 & 0,06 & $-0,60$ \\
\hline & $\mathrm{LS}(\mathrm{mm})$ & 6,74 & 13,95 & 9,55 & 1,55 & 16,23 & 0,89 & 0,67 \\
\hline & $\mathrm{ES}(\mathrm{mm})$ & 0,44 & 1,48 & 0,86 & 0,01 & 1,16 & 0,44 & $-0,60$ \\
\hline & MFS (mg) & 18,00 & 62,00 & 32,05 & 9,38 & 29,27 & 1,17 & 1,55 \\
\hline \multirow{4}{*}{ 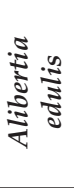 } & $\mathrm{CS}(\mathrm{mm})$ & 3,67 & 6,29 & 5,01 & 0,50 & 9,98 & $-0,12$ & 0,20 \\
\hline & $\mathrm{LS}(\mathrm{mm})$ & 2,19 & 4,15 & 3,24 & 0,50 & 15,43 & $-0,21$ & $-0,87$ \\
\hline & $\mathrm{ES}(\mathrm{mm})$ & 1,06 & 2,39 & 1,58 & 0,26 & 16,46 & 0,45 & 0,81 \\
\hline & MFS (mg) & 6,40 & 24,10 & 13,84 & 4,18 & 31,01 & 0,63 & $-0,01$ \\
\hline \multirow{4}{*}{ 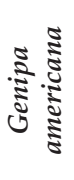 } & $\mathrm{CS}(\mathrm{mm})$ & 5,53 & 9,53 & 7,11 & 0,88 & 12,38 & 0,77 & 0,20 \\
\hline & $\mathrm{LS}(\mathrm{mm})$ & 4,17 & 7,86 & 5,82 & 0,74 & 12,71 & 0,51 & 0,68 \\
\hline & $\mathrm{ES}(\mathrm{mm})$ & 1,13 & 2,46 & 1,67 & 0,27 & 16,17 & 0,28 & 0,16 \\
\hline & MFS (mg) & 23,30 & 64,50 & 37,57 & 8,96 & 23,85 & 0,53 & 1,84 \\
\hline
\end{tabular}

CS = Comprimento da semente, LS = largura da semente, ES = espessura da semente e MFS = Matéria fresca da semente. 
das sementes de $A$. edulis apresentaram valores positivos, indicando que as sementes com menor massa e tamanho predominaram na amostra analisada. O comprimento e largura da semente de $A$. edulis apresentaram coeficiente de assimetria negativo, indicando que na amostra analisada predominaram as sementes com maior comprimento e largura. O coeficiente de curtose foi menor que $3(K<3)$ para todas as características das três espécies em estudo, indicando que a distribuição de frequência das características avaliadas é mais achatada que a curva normal, ou seja, apresentam maior amplitude de distribuição dos dados.

As correlações entre as características dos frutos das três espécies foram todas positivas e significativas, exceto para as correlações entre número de sementes por fruto com a massa da matéria fresca, comprimento e espessura dos frutos de L. pacari. As correlações formadas pelas características das sementes com as dos frutos foram positivas e outras negativas, mas nenhuma foi significativa. O mesmo também se observou para as correlações geradas das características das sementes, com exceção para a correlação da massa da matéria fresca da semente com seu comprimento em L. pacari, e a correlação da espessura e massa da matéria fresca com o comprimento da semente de G. americana, que foram positivas e significativas (Quadro 3).

Foram consideradas correlações fortes $(0,8 \leq \mathrm{P}<1)$, conforme Santos (2010), as que ocorreram entre massa a fresca da semente e seu comprimento em L. pacari, diâmetro transversal e longitudinal com a massa da matéria fresca dos frutos de A. edulis,

Quadro 3 - Matriz de coeficientes de correlações de Pearson das características do fruto e da semente de três espécies colhidos no município de Cáceres/MT

\begin{tabular}{|c|c|c|c|c|c|c|c|c|c|}
\hline & & MFF & $\mathrm{CF}$ & $\mathrm{EF}$ & LF & NSF & CS & LS & ES \\
\hline \multirow{9}{*}{ 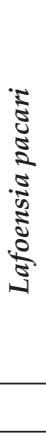 } & $\mathrm{CF}$ & $0,692^{* *}$ & & & & & & & \\
\hline & $\mathrm{EF}$ & $0,648^{* *}$ & $0,600^{* *}$ & & & & & & \\
\hline & $\mathrm{LF}$ & $0,784^{* *}$ & $0,573^{* *}$ & $0,606^{* *}$ & & & & & \\
\hline & NSF & $0,173^{\mathrm{ns}}$ & $0,027^{\mathrm{ns}}$ & $0,208^{\mathrm{ns}}$ & $0,288^{* *}$ & & & & \\
\hline & CS & $0,048^{\mathrm{ns}}$ & $-0,057 \mathrm{~ns}$ & $0,091 \mathrm{~ns}$ & 0,039 ns & $0,036^{\text {ns }}$ & & & \\
\hline & LS & $-0,034^{\mathrm{ns}}$ & $0,126^{\mathrm{ns}}$ & $-0,041^{\mathrm{ns}}$ & $0,000^{\mathrm{ns}}$ & $-0,033^{\mathrm{ns}}$ & 0,069 ns & & \\
\hline & ES & $0,054^{\mathrm{ns}}$ & $0,040^{\mathrm{ns}}$ & $-0,019 \mathrm{~ns}$ & $-0,013^{\mathrm{ns}}$ & $0,103^{\text {ns }}$ & $-0,121^{\mathrm{ns}}$ & $0,019 \mathrm{~ns}$ & \\
\hline & MFS & $-0,021^{\mathrm{ns}}$ & $-0,087^{\mathrm{ns}}$ & $0,017^{\mathrm{ns}}$ & $-0,003^{\text {ns }}$ & $-0,003^{\text {ns }}$ & $0,946^{* *}$ & $0,040^{\mathrm{ns}}$ & $-0,007^{\mathrm{ns}}$ \\
\hline & & MFF & DLF & DTF & NSF & $\mathrm{CS}$ & LS & ES & \\
\hline \multirow{8}{*}{ 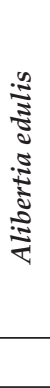 } & DLF & $0,811^{* *}$ & & & & & & & \\
\hline & DTF & $0,967^{* *}$ & $0,701^{* *}$ & & & & & & \\
\hline & NSF & $0,761^{* *}$ & $0,566^{* *}$ & $0,743^{* *}$ & & & & & \\
\hline & CS & $0,039^{\text {ns }}$ & $0,053^{\mathrm{ns}}$ & $0,026^{\mathrm{ns}}$ & $0,111^{\mathrm{ns}}$ & & & & \\
\hline & LS & $0,203^{\text {ns }}$ & $0,110^{\mathrm{ns}}$ & $0,262^{*}$ & 0,239 ns & $-0,128$ & & & \\
\hline & ES & $-0,068^{\text {ns }}$ & $-0,147^{\mathrm{ns}}$ & $-0,080^{\mathrm{ns}}$ & $0,091^{\text {ns }}$ & $-0,069$ & $-0,004^{\mathrm{ns}}$ & & \\
\hline & MFS & $0,005^{\mathrm{ns}}$ & $-0,122^{\mathrm{ns}}$ & $0,011^{\mathrm{ns}}$ & $0,153^{\text {ns }}$ & $0,139^{\text {ns }}$ & $-0,076^{\mathrm{ns}}$ & $-0,095^{\mathrm{ns}}$ & \\
\hline & & MFF & DLF & DTF & NSF & CS & LS & ES & \\
\hline \multirow{7}{*}{ 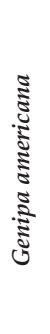 } & DLF & $0,828^{* *}$ & & & & & & & \\
\hline & DTF & $0,899^{* *}$ & $0,674^{* *}$ & & & & & & \\
\hline & NSF & $0,588^{* *}$ & $0,515^{* *}$ & $0,575^{* *}$ & & & & & \\
\hline & CS & $0,050^{\mathrm{ns}}$ & $-0,017^{\mathrm{ns}}$ & $0,012^{\text {ns }}$ & $-0,018^{\mathrm{ns}}$ & & & & \\
\hline & LS & $-0,159^{\mathrm{ns}}$ & $-0,178^{\mathrm{ns}}$ & $-0,180^{\mathrm{ns}}$ & $-0,158^{\mathrm{ns}}$ & $0,005^{\mathrm{ns}}$ & & & \\
\hline & ES & $-0,221$ ns & $0,211^{\mathrm{ns}}$ & $0,254^{\mathrm{ns}}$ & $0,084^{\mathrm{ns}}$ & $-0,452^{* *}$ & $-0,116^{\mathrm{ns}}$ & & \\
\hline & MFS & $0,087^{\mathrm{ns}}$ & $0,132^{\mathrm{ns}}$ & $-0,014^{\mathrm{ns}}$ & $-0,037 \mathrm{~ns}$ & $-0,322^{*}$ & $-0,030^{\mathrm{ns}}$ & $-0,021 \mathrm{~ns}$ & \\
\hline
\end{tabular}

** , * - Significativo a $1 \%$ e $5 \%$ de probabilidade, respectivamente, pelo teste $\mathrm{t}$. 
tendo o mesmo também ocorrido em G. americana, sendo todas elas significativas. As correlações entre a massa de matéria fresca do fruto com sua espessura e largura, do seu comprimento com sua espessura e desta com a largura do mesmo em $L$. pacari foram classificadas como moderadas $(0,5 \leq \mathrm{P}<0,8)$. O mesmo também se verificou para as correlações massa da matéria fresca do fruto de A. edulis com o número de sementes, do diâmetro transversal com o longitudinal e com o número de sementes, já em G. americana a única correlação moderada foi entre o diâmetro transversal e longitudinal do fruto.

Os resultados obtidos servem para demonstrar que em espécies não domesticadas, como é o caso das utilizadas neste estudo, é comum que ocorram variações das características morfométricas, como foi constatado em sementes de Jacaranda decurrens subsp. symmetrifoliolata por Sangalli et al. (2012), de Erythrina velutina por Silva Junior et al. (2012) e de Dipteryx alata Vog. por Zuffo et al. (2014). As oscilações ocorridas nas características biométricas das sementes podem ser oriundas de dois fatores, os quais podem agir de forma isolada ou em conjunto, sendo estes fatores de origem genética ou ambiental (Nogueira et al., 2010).

O conhecimento da variação biométrica de caracteres de frutos e sementes é importante para criação de bancos de germoplasma e para o melhoramento dessas características, seja no sentido de aumento ou uniformidade (Gonçalves et al., 2013).

\section{CONCLUSÕES}

Nos frutos a maior variação ocorre na característica massa da matéria fresca em $L$. pacari e no número de sementes por fruto em $A$. edulis e G. americana. Nas sementes isso ocorre na massa da matéria fresca.

A maioria das características biométricas dos frutos apresenta coeficiente de assimetria negativo, enquanto nas sementes a maioria apresenta coeficiente de assimetria positivo. O coeficiente de curtose, para todas as características biométricas dos frutos e sementes apresentam valor menor que 3, exceto para comprimento do fruto de L. pacari.

Verifica-se correlação significativa e positiva dentre as características do fruto, exceto para número de sementes por fruto com a massa da matéria fresca, comprimento e espessura nos frutos de L. pacari, enquanto para as sementes não há correlação significativa, exceto massa da matéria fresca com o comprimento em L. pacari e do comprimento da semente com a espessura e matéria fresca em G. americana.

\section{REFERÊNCIAS BIBLIOGRÁFICAS}

Almeida, S.P.; Silva, J.A. \& Ribeiro, J.F. (1987) - Aproveitamento alimentar de espécies nativas dos cerrados: araticum, baru, cagaita e jatobá. Planaltina, Embrapa-CPAC. 83p. (Documentos 26).

Araújo, E.C.; Mendonça, A.V.R.; Barroso, D.G.; Lamônica, K.R. \& Silva, R.F. (2004) - Caracterização morfológica de frutos, sementes e plântulas de Sesbania virgata (Cav.) Pers. Revista Brasileira de Sementes, vol. 26, n. 1, p. 105-110. http://dx.doi.org/10.1590/S0101-31222004000100016

Baskin, C.S. \& Baskin, J.M. (1998) - Seeds: ecology, biogeography, and evolution of dormancy and germination. London, Academic Press, 666 p.

Battilani, J.L.; Santiago, E.F. \& Dias, E.S. (2011) - Morfologia de frutos, sementes, plântulas e plantas jovens de Guibourtia hymenifolia (Moric.) J. Leonard (Fabaceae). Revista Árvore, vol. 35, n. 5, p. 1089-1098. http:// dx.doi.org/10.1590/S0100-67622011000600015

Brasil. (2009) - Regras para análise de sementes. Brasília, Mapa/ACS, 395p.

Brüning, F.O.; Lúcio, A.D. \& Muniz, M.F.B. (2011) - Padrões para germinação, pureza, umidade e peso de mil sementes em análises de sementes de espécies florestais nativas do Rio Grande do Sul. Ciência Florestal, vol. 21, n. 2, p. 193-202. http://dx.doi.org/10.5902/198050983221

Carvalho, P.E.R. (2003a) - Espécies arbóreas brasileiras. Brasília, Embrapa, p. 441-448, vol. 1

Carvalho, P.E.R. (2003b) - Jenipapeiro. Colombo, Embrapa Floresta, 14 p. (Circular Técnica, 80).

Donadio, L.C.; Nachtigal, J.C. \& Sacramento, C.K. (1998) - Frutas exóticas. Jaboticabal, Funep, 278 p. 
Figueiredo, R.W.; Maia, G.A.; Monteiro, J.C.S. \& Figueiredo, E.A.T. (1991) - Composição de ácidos graxos na fração lipídica de polpa e semente de jenipapo (Genipa americana L.). Boletim do Centro de Pesquisa de Processamento de Alimentos, vol. 9, n. 2, p. 149-154. http://dx.doi.org/10.5380/cep.v9i2.14449

Gonçalves, L.G.V.; Andrade, F.R.; Marimon Junior, B.H.; Schossler, T.R.; Lenza, E. \& Marimon, B.S. (2013) - Biometria de frutos e sementes de mangaba (Hancornia speciosa Gomes) em vegetação natural na região leste de Mato Grosso, Brasil. Revista de Ciências Agrárias, vol. 36, n. 1, p. 31-40.

Gusmão, E.; Vieira, F.A. \& Fonseca Júnior, E.M. (2006) - Biometria de frutos e endocarpos de murici (Byrsonima verbascifolia Rich. ex A. Juss.). Cerne, vol. 12, n. 1, p. 84-91.

Marcos Filho, J. (2015) - Fisiologia de sementes de plantas cultivadas. Londrina, Abrates, 659 p.

Martin, F.W.; Campbell, C.W. \& Ruberté, R.M. (1987) - Perennia edible fruits of the tropics: an inventory. Washington, Department of agriculture handbook, n. 642, 252 p.

Mendonça, R.C.; Felfili, J.M.; Walter, B.M.T.; Silva Júnior, M.C.; Rezende, A.V.; Filgueiras, T.S.; Nogueira, P.E. \& Fagg, C.W. (2008) - Flora vascular do bioma Cerrado - Checklist com 12.356 espécies. In: Sano, S.M.; Almeida, S.P. \& Ribeiro, J.F. (Eds.) - Cerrado: ambiente e flora. Planaltina, Embrapa, p. 421-443, vol. 2.

Moura, R.C.; Lopes, P.S.N.; Brandão Junior, D.S.; Gomes, J.G. \& Pereira, M.B. (2010) - Biometria de frutos e sementes de Butia capitata (Mart.) Beccari (Arecaceae), em vegetação natural no Norte de Minas Gerais, Brasil. Biota Neotropica, vol. 10, n. 2, p. 415-419. http://dx.doi.org/10.1590/S1676-06032010000200040

Moura, N.F.; Chaves, L.J. \& Naves, R.V. (2013) - Caracterização física de frutos de pequizeiro (Caryocar brasiliense Camb) do cerrado. Revista Árvore, vol. 37, n. 5, p. 905-912. http://dx.doi.org/10.1590//0100-67622013000500013

Naves, R.V.; Almeida Neto, J.X.; Rocha, M.R.; Borges, J.D.; Carvalho, G.C.; Chaves, L.J. \& Silva, V.A. (1995) - Determinação de características físicas em frutos e teor de nutrientes, em folhas e no solo, de três espécies frutíferas de ocorrência natural nos cerrados de Goiás. Anais da Escola de Agronomia e Veterinária, vol. 25, n. 2, p. 107-114.

Nogueira, F.C.B.; Medeiros Filho, S. \& Gallão, M.I. (2010) - Caracterização da germinação e morfologia de frutos, sementes e plântulas de Dalbergia cearenses Ducke (pau-violeta) - Fabaceae. Acta Botanica Brasilica, vol. 24, n. 4, p. 978-985. http://dx.doi.org/10.1590/50102-33062010000400013

R Core Team. (2012) - R: A language and environment for statistical computing. Viena, R Foundation for Statistical Computing.

Sangalli, A.; Vieira, M.C.; Scalon, S.P.Q.; Zárate, N.A.H.; Silva, C.B. \& Ribeiro, I.S. (2012) - Morfometria de frutos e sementes e germinação de carobinha (Jacaranda decurrens subsp. symmetrifoliolata Farias \& Proença), após o armazenamento. Revista Brasileira de Plantas Medicinais, vol. 14, n. 2, p. 267-275. http:// dx.doi.org/10.1590/S1516-05722012000200003

Santos, C. (2010) - Estatística descritiva: manual de auto-aprendizagem. Lisboa, Silabo, 264 p.

Santos, F.S.; Paula, R.C.; Sabonaro, D.Z. \& Valadares, J. (2009b) - Biometria e qualidade fisiológica de sementes de diferentes matrizes de Tabebuia chrysotricha (Mart. Ex A. DC.) StandI. Scientia Forestalis, vol. 37, n. 82, p. 163-173.

Santos, L.W.; Coelho, M.F.B. \& Pirani, F.R. (2009a) - Fenologia de Lafoensia pacari A.St.-Hil. (Lythraceae) em Barra do Garças, Mato Grosso, Brasil. Revista Brasileira de Plantas Medicinais, vol. 11, n. 1, p. 12-17. http:// dx.doi.org/10.1590/S1516-05722009000100003

Silva Júnior, M.C. (2005) - 100 Árvores do cerrado: guia de campo. Brasília, Rede de sementes do cerrado, 278 p. Silva Junior, V.T.; Lima, J.M.G.M.; Rodrigues, C.W.M.S. \& Barbosa, D.C.A. (2012) - Erythrina velutina willd. (Leguminosae-Papilionoideae) ocorrente em caatinga e brejo de altitude de Pernambuco: biometria, embebição e germinação. Revista Árvore, vol. 36, n. 2, p. 247-257. http://dx.doi.org/10.1590/S0100-67622012000200006

Silva, D.B.; Silva, J.A.; Junqueira, N.T.V. \& Andrade, L.R.M. (2001) - Frutas do Cerrado. Brasília, Embrapa Cerrados, $179 \mathrm{p}$.

Vieira, F.A. \& Gusmão, E. (2008) - Biometria, armazenamento de sementes e emergência de plântulas de Talisia esculenta Radlk. (Sapindaceae). Ciência e Agrotecnolgia, vol. 32, n. 4, p. 1073-1079. http://dx.doi. org/10.1590/S1413-70542008000400006

Zuffo, A.M.; Andrade, F.R. \& Zuffo Junior, J.M. (2014) - Caracterização biométrica de frutos e sementes de baru (Dipteryx alata Vog.) na região leste de Mato Grosso, Brasil. Revista de Ciências Agrárias, vol. 37, n. 4, p. $463-471$. 